\title{
Main complications and results of treatment with intra-arterial infusion chemotherapy through the subclavian and thoracic arteries for locally advanced breast cancer
}

\author{
XIAOYI WANG ${ }^{1}$, CHANGING GAN $^{1}$, HONGYUAN LI ${ }^{1}$, YUXIAN WEI ${ }^{1}$, DONCHANG ZHU ${ }^{1}$, \\ GUANGLUN YANG ${ }^{1}$, XINLIANG SU ${ }^{1}$, JEAN-FRANÇOIS RODIER ${ }^{2}$ and GUOSHENG REN ${ }^{1}$ \\ ${ }^{1}$ Department of Breast and Endocrine Surgery, First Affiliated Hospital, Chongqing Medical University, Chongqing 400016, \\ P.R. China; ${ }^{2}$ Department of Surgical Oncology, Paul Strauss Cancer Center, 67065 Strasbourg, France
}

Received November 11, 2012; Accepted April 16, 2013

DOI: $10.3892 / \operatorname{mco} .2013 .129$

\begin{abstract}
Intra-arterial infusion chemotherapy for locally advanced breast cancer (LABC) has been previously performed. However, the main complications of this type of chemotherapy remain to be clarified. In the present study, catheterization chemotherapy was carried out for 53 LABC cases (stage IIIa-IIIc) between May, 2006 and March, 2007. For IIIB and IIIC patients, the catheters were guided to the opening of the subclavian artery. For stage IIIa patients, the catheters were placed into the thoracic artery through a subcutaneous femoral artery puncture. One to four cycles of chemotherapy (mean, 1.6 cycles) were administered for the patients using taxotere, epidoxorubicin, 5-fluorouracil and/or cyclophosphamide. The interval time between the two cycles was 21 days. Seven cases were identified as complete response (CR, 13.2\%), 41 cases were partial response (PR, 77.4\%) with a rate of effectiveness of $(\mathrm{CR}+\mathrm{PR}, 90.6 \%), 5$ cases were stable disease $(\mathrm{SD}, 9.40 \%)$ and no case was progressive. Pain of the ipsilateral upper extremity was present in 7 cases. Two cases exhibited ipsilateral upper extremity atrophy following drug administration from the opening of the subclavian artery. One case experienced neck pain and headache, while in one case necrosis of local skin was evident. Hematological toxicity over grade 3 was observed in 6 cases $(11.30 \%)$. Systemic toxicity was mild and did not affect the quality of life of the patients. Overall survival was identified as $18 / 51(35.3 \%)$, and free-disease survival as 10/51 (19.6\%). In conclusion, intra-arterial infusion chemotherapy is an effective local control treatment for LABC. The main complications are pain of the ipsilateral upper extremity and neck as well as headache. Severe complications are ipsilateral
\end{abstract}

Correspondence to: Professor Guosheng Ren, Department of Breast and Endocrine Surgery, First Affiliated Hospital, Chongqing Medical University, 1 You Yi Road, Chongqing 400016, P.R. China E-mail:rgs726@163.com

Key words: locally advanced breast cancer, efficacy and complication, intra-arterial chemotherapy, subclavian artery, thoracic artery upper extremity atrophy and necrosis of local skin. During the treatment, controlling the pressure of the tourniquet and velocity of drug administration are crucial for reducing local complications.

\section{Introduction}

Locally advanced breast cancer (LABC) is defined as patients who present with the clinical stages of T4 N0-1M0 and T3N1 (stage IIIa), T0-3 N2M0 (stage IIIb), and T0-4 N3M0 (stage IIIc), without distant metastasis. $\mathrm{T}$ and $\mathrm{N}$ staging were as per the 6th edition of the American Joint Committee on Cancer, the TNM staging classification and revision of the American Joint Committee on Cancer staging system for breast cancer (1). LABC is characterized by large breast tumors involving the skin or muscles of the chest wall and extensive involvement of the local lymph nodes. Therefore treatment involves combined modality including systemic chemotherapy, surgery and radiotherapy $(2,3)$.

Good local control and down-staging increase the possibility for surgery. Intra-arterial infusion is suggested to take advantage of the first pass effect of chemotherapeutics, generating higher local drug concentrations at the tumor cell membrane and therefore enhancing cellular drug uptake. Drug exposure to the tumor starts at the time of drug uptake through the cell membrane. Studies have focused on intra-arterial infusion chemotherapy for LABC and results have demonstrated high local control (4-9). However, the main complications of intra-arterial infusion chemotherapy have yet to be reported. In the present study, we report on the results and main complications of intra-arterial infusion chemotherapy through the subclavian and thoracic arteries for $53 \mathrm{LABC}$ cases for the period December, 2006 to December, 2010. The results demonstrated the efficacy of good local control for LABC and revealed serious complications with intra-arterial infusion chemotherapy.

\section{Materials and methods}

Subjects. The patients with LABC were recruited from the Department of Breast and Endocrine Surgery, The First 
Affiliated Hospital of Chongqing Medical University, between December, 2006 and December, 2010. Clinical stage IIIa was identified in 10 patients, stage IIIb in 24 patients and stage IIIc in 19 patients. The patients were female with a mean age of 47.7 years (range, 28-67 years). Histological examination confirmed carcinoma of the breast (left side in 30 and right side in 23) by pathological diagnosis via fine needle biopsy pre-operative intra-arterial infusion chemotherapy. The findings of the chest X-ray, ventral ultrasonography, radioisotope scan and head nuclear magnetic resonance did not provide evidence of distant metastasis. This study was approved by the Ethics Committee of Chongqing Medical University. All patients provided written consent.

Treatment. The patients were treated with angiography (Siemens Coroskop Plus, Germany) under local anesthesia. According to the Seldinger technique, a $6 \mathrm{~F}$ catheter was inserted percutaneously into the femoral artery. Subsequently, the catheter was guided to the opening of the subclavian artery. An arteriogram of the subclavian, thoracic and vertebral arteries was obtained for the patients (Fig. 1). For patients in stage IIIa, the catheter was inserted into the thoracic artery (Fig. 2). For patients in stage IIIb and IIIc, the catheter was guided to the opening of the subclavian and lateral thoracic arteries (Fig. 1). Prior to drug administration, contrast agent (iohexol) was injected to comfirm the location of the catheter again and the ipsilateral upper arm was bundled with a tourniquet, at a pressure of $260-280 \mathrm{mmHg}$.

Drug doses administered included taxotere $\left(100 \mathrm{mg} / \mathrm{m}^{2}\right)$, epidoxorubicin $\left(100 \mathrm{mg} / \mathrm{m}^{2}\right), 5$-fluorouracil $(1,000 \mathrm{mg})$ and/or cyclophosphamide $\left(800 \mathrm{mg} / \mathrm{m}^{2}\right)$. The tumor response, local lymph nodes and occurrence of local or systemic complications determined the number of cycles of chemotherapy for which there was an interval time of 21 days between two cycles.

Response criteria. Based on the WHO criteria the response was estimated according to the clinical features following treatment and all the patients were evaluated by computerised tomography scan preoperation. Complete disappearance of all the lesions was considered a complete response (CR); macroscopic reduction in size by $\geq 50 \%$ was considered a partial response (PR); a reduction of $25-50 \%$ was designated as stable disease (SD); and the appearance of any new lesions not previously identified or an estimated increase of $25 \%$ in existent lesions was considered progressive disease.

\section{Results}

Of the 53 patients, 7 cases (13.2\%) were CR; 41 cases (77.4\%) were PR, with a rate of effectiveness of (CR + PR: 90.6\%,48/53); 5 cases $(9.4 \%)$ were SD and no case was progressive. The treatment results of LABC are provided in Table I.

Main complications. Complications occurred mainly in the local areas. Pain of the ipsilateral upper extremity was noted in 7 cases. One case experienced neck pain and headache; this patient recovered 2 weeks later without any special treatment. Two cases had ipsilateral upper extremity atrophy and disability and did not recover within a time period of 6 months, following drug administration from the opening

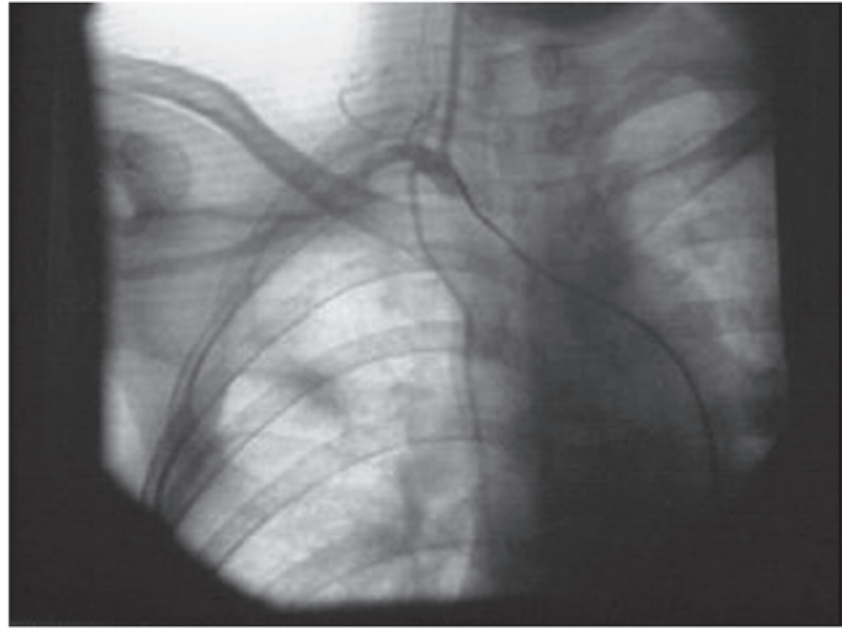

Figure 1. For patients in stage IIIb and IIIc, the catheter was guided to the opening of the subclavian and lateral thoracic arteries. Prior to drug administration, a contrast agent (iohexol) was injected to comfirm the location of the catheter. An arteriogram of the subclavian, thoracic and vertebral arteries was obtained.

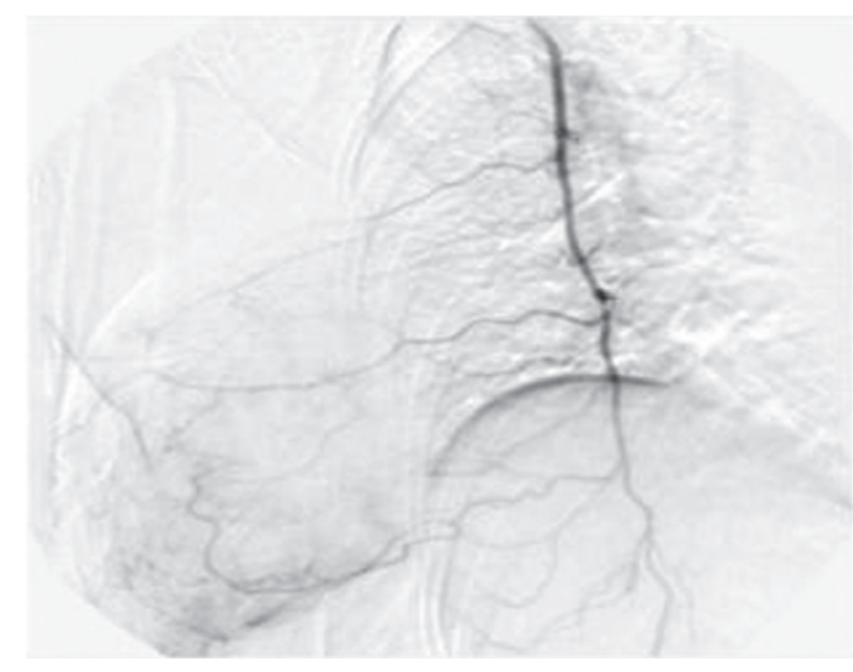

Figure 2. Arteriogram of the thoracic artery. For patients in stage IIIa, the catheter was inserted into the thoracic artery. Prior to drug administration, contrast agent (iohexol) was injected to comfirm the location of the catheter.

of the subclavian and lateral thoracic arteries. One case had necrosis of local skin, but recovered with conservative treatment. The systemic toxicity was mild and did not affect the quality of life of patients (Table I).

Other complications. Of the 53 patients, no complications related to the angiographic technique were observed. Hematological toxicity over grade 3 such as fever and without bleeding was observed in 6 cases $(11.3 \%$ ), while 9 patients had gastrointestinal symptoms including nausea, vomiting, diarrhea and stomachache. Cardiovascular toxicity was not observed (Table I).

\section{Discussion}

At present, a combination of systemic therapy with locoregional treatment (surgery and/or radiotherapy) constitutes the 
Table I. Patient characteristics, treatment, response and complications.

\begin{tabular}{|c|c|c|c|c|c|c|c|}
\hline No. & Age (years) & Stage & Location & Cycles & Drugs & Response & Complication \\
\hline 1 & 32 & $\mathrm{IIIb}$ & Left & 2 & EPI & PR & No \\
\hline 2 & 28 & IIIb & Right & 1 & $\mathrm{EPI}+\mathrm{CTX}+5-\mathrm{FU}$ & SD & Inappetence \\
\hline 3 & 53 & IIIb & Right & 1 & $\mathrm{EPI}+\mathrm{CTX}+5-\mathrm{FU}$ & PR & No \\
\hline 4 & 67 & $\mathrm{IIIb}$ & Left & 1 & EPI & PR & Pain of upper extremity \\
\hline 5 & 65 & IIIb & Left & 3 & $\mathrm{EPI}+\mathrm{CTX}+5-\mathrm{FU}$ & CR & Inappetence \\
\hline 6 & 52 & $\mathrm{IIIb}$ & Right & 1 & $\mathrm{EPI}+5-\mathrm{FU}$ & PR & No \\
\hline 7 & 51 & IIIa & Left & 1 & $\mathrm{EPI}+\mathrm{CTX}+5-\mathrm{FU}$ & PR & No \\
\hline 8 & 47 & IIIa & Right & 1 & $\mathrm{EPI}+\mathrm{CTX}+5-\mathrm{FU}$ & PR & No \\
\hline 9 & 28 & IIIa & Left & 2 & $\mathrm{~T}$ & PR & Hematological toxicity over grade 3 \\
\hline 10 & 32 & $\mathrm{IIIb}$ & Left & 4 & $\mathrm{~T}$ & $\mathrm{CR}$ & No \\
\hline 11 & 57 & IIIa & Right & 1 & $\mathrm{EPI}+\mathrm{CTX}+5-\mathrm{FU}$ & SD & Pain of upper extremity \\
\hline 12 & 67 & IIIc & Left & 4 & $\mathrm{~T}$ & $\mathrm{CR}$ & Hematological toxicity over grade 3 \\
\hline 13 & 31 & IIIa & Left & 1 & $\mathrm{EPI}+\mathrm{CTX}+5-\mathrm{FU}$ & PR & No \\
\hline 14 & 34 & IIIc & Right & 1 & $\mathrm{EPI}+\mathrm{CTX}+5-\mathrm{FU}$ & PR & No \\
\hline 15 & 51 & IIIa & Left & 1 & $\mathrm{~T}$ & PR & No \\
\hline 16 & 66 & IIIc & Right & 1 & $\mathrm{~T}$ & PR & Pain of upper extremity \\
\hline 17 & 56 & IIIc & Right & 2 & $\mathrm{~T}$ & PR & Necrosis of local skin \\
\hline 18 & 41 & IIIa & Left & 1 & $\mathrm{~T}$ & PR & No \\
\hline 19 & 46 & IIIc & Right & 2 & $\mathrm{EPI}+\mathrm{CTX}+5-\mathrm{FU}$ & PR & No \\
\hline 20 & 39 & IIIc & Left & 2 & $\mathrm{~T}$ & PR & No \\
\hline 21 & 41 & IIIb & Left & 2 & $\mathrm{EPI}+\mathrm{CTX}+5-\mathrm{FU}$ & PR & No \\
\hline 22 & 45 & IIIa & Left & 1 & $\mathrm{EPI}+\mathrm{CTX}+5-\mathrm{FU}$ & PR & Pain of upper extremity \\
\hline 23 & 50 & IIIb & Right & 1 & $\mathrm{~T}$ & PR & Pain of upper extremity; nausea \\
\hline 24 & 50 & IIIb & Left & 2 & $\mathrm{EPI}+\mathrm{CTX}+5-\mathrm{FU}$ & PR & No \\
\hline 25 & 50 & IIIc & Left & 1 & $\mathrm{EPI}+\mathrm{CTX}+\mathrm{T}$ & PR & No \\
\hline 26 & 49 & IIIb & Left & 2 & $\mathrm{EPI}+\mathrm{CTX}+\mathrm{T}$ & $\mathrm{CR}$ & Pain of upper extremity \\
\hline 27 & 48 & IIIb & Left & 2 & EPI+CTX+T & PR & Diarrhea \\
\hline 28 & 43 & $\mathrm{IIIb}$ & Right & 1 & $\mathrm{EPI}+\mathrm{CTX}+5-\mathrm{FU}$ & PR & No \\
\hline 29 & 36 & IIIc & Left & 1 & $\mathrm{EPI}+\mathrm{CTX}+5-\mathrm{FU}$ & PR & Nausea \\
\hline 30 & 36 & IIIb & Right & 2 & $\mathrm{EPI}+\mathrm{CTX}+\mathrm{T}$ & PR & Hematological toxicity over grade 3 \\
\hline 31 & 54 & IIIb & Left & 1 & $\mathrm{EPI}+\mathrm{CTX}+\mathrm{T}$ & PR & No \\
\hline 32 & 60 & IIIc & Right & 2 & $\mathrm{EPI}+\mathrm{CTX}+\mathrm{T}$ & $\mathrm{CR}$ & Pain of upper extremity \\
\hline 33 & 66 & IIIc & Right & 1 & $\mathrm{EPI}+\mathrm{CTX}+\mathrm{T}$ & PR & Diarrhea \\
\hline 34 & 53 & IIIc & Left & 1 & $\mathrm{EPI}+\mathrm{CTX}+\mathrm{T}$ & PR & No \\
\hline 35 & 42 & IIIc & Right & 1 & $\mathrm{EPI}+\mathrm{CTX}+\mathrm{T}$ & PR & No \\
\hline 36 & 42 & IIIb & Right & 1 & $\mathrm{EPI}+\mathrm{CTX}+\mathrm{T}$ & PR & Stomachache \\
\hline 37 & 42 & IIIa & Right & 2 & $\mathrm{EPI}+\mathrm{CTX}+\mathrm{T}$ & PR & No \\
\hline 38 & 47 & IIIb & Left & 2 & $\mathrm{EPI}+\mathrm{CTX}+5-\mathrm{FU}$ & SD & No \\
\hline 39 & 45 & IIIb & Right & 3 & $\mathrm{EPI}+\mathrm{CTX}+\mathrm{T}$ & $\mathrm{CR}$ & Ipsilateral upper extremity atrophy \\
\hline 40 & 29 & $\mathrm{IIIb}$ & Right & 2 & $\mathrm{EPI}+\mathrm{CTX}+\mathrm{T}$ & PR & Hematological toxicity over grade 3 \\
\hline 41 & 53 & IIIc & Left & 2 & $\mathrm{EPI}+\mathrm{CTX}+\mathrm{T}$ & PR & Nausea \\
\hline 42 & 29 & IIIb & Right & 2 & $\mathrm{EPI}+\mathrm{CTX}+\mathrm{T}$ & PR & No \\
\hline 43 & 60 & IIIc & Left & 1 & $\mathrm{EPI}+\mathrm{CTX}+\mathrm{T}$ & PR & No \\
\hline 44 & 61 & IIIc & Left & 3 & $\mathrm{EPI}+\mathrm{CTX}+\mathrm{T}$ & PR & Hematological toxicity over grade 3 \\
\hline 45 & 60 & IIIc & Left & 1 & $\mathrm{EPI}+\mathrm{CTX}+5-\mathrm{FU}$ & SD & No \\
\hline 46 & 52 & $\mathrm{IIIb}$ & Left & 2 & $\mathrm{EPI}+\mathrm{CTX}+5-\mathrm{FU}$ & PR & Pain of neck and headache \\
\hline 47 & 47 & $\mathrm{IIIb}$ & Left & 1 & $\mathrm{EPI}+\mathrm{CTX}+5-\mathrm{FU}$ & SD & No \\
\hline 48 & 61 & IIIc & Left & 2 & $\mathrm{EPI}+\mathrm{CTX}+\mathrm{T}$ & PR & No \\
\hline 49 & 62 & IIIc & Right & 1 & $\mathrm{~T}$ & PR & No \\
\hline 50 & 54 & $\mathrm{IIIb}$ & Right & 1 & $\mathrm{EPI}+\mathrm{CTX}+\mathrm{T}$ & PR & No \\
\hline 51 & 42 & IIIb & Right & 2 & $\mathrm{EPI}+\mathrm{CTX}+\mathrm{T}$ & PR & Hematological toxicity over grade 3 \\
\hline 52 & 55 & IIIa & Left & 2 & $\mathrm{EPI}+\mathrm{CTX}+\mathrm{T}$ & $\mathrm{CR}$ & Vomiting \\
\hline 53 & 60 & $\mathrm{IIIb}$ & Left & 1 & $\mathrm{EPI}+\mathrm{CTX}+\mathrm{T}$ & PR & Ipsilateral upper extremity atrophy \\
\hline
\end{tabular}


standard of care in LABC patients since improving locoregional control is associated with improved survival (10). In patients with stage III breast cancer treated with induction chemotherapy followed by surgery, radiotherapy or combination therapy, the risk of locoregional recurrence is at a range of $20 \%$ (9). The use of induction systemic therapy results in tumor downstaging and in selected LABC patients even allows for breast conserving surgery (11-14).

Intra-arterial infusion chemotherapy is an effective and safe treatment for the local tumor control of LABC (7). In our data, which also demonstrated the good local control of LABC with intra-arterial infusion chemotherapy, the CR + PR was $90.6 \%$, which was higher than that reported by Shimamoto et al (8), who noted that the local response rate was $77.3 \%$ (at least more than two regimens) and Pacetti et al (7) who noted that the response rate was $80 \%$. Factors such as the drugs used and administration of chemotheraputic cycles likely affected the results of those authors. However, in the present study, treatment involved different methods of drug administration. For patients in stage IIIa, large breast tumors and the skin or muscles of the chest wall were usually involved, thus local control tumors were primary tumors. We inserted a catheter into the thoracic artery and administered the majority of the drugs into the chest area, as there would be more effective local control and downstaging. For patients in stage IIIb and IIIc, the local lymph nodes were usually extensively involved. Control of the regional lymph nodes was considered crucial, therefore, the catheter was guided to the opening of the subclavian and lateral thoracic arteries, allowing more drugs to be administered in the subclavian, superclavian and axilla regions These methods contributed to improving the response rate of local lesions and increased the possibility of surgery. However, results of the follow-up revealed that overall and disease-free survival had not improved.

Few studies have reported on local complications following intra-arterial infusion chemotherapy for LABC. In the present study, severe complications were observed during treatment. Two patients in stage IIIb had ipsilateral upper extremity atrophy leading to disability, and these patients did not recover within a 6-month time period. Pain of the ipsilateral upper extremity was noted in 7 cases that recovered two weeks later without any special treatment. The reasons for these complications included the loosening of the tourniquet during drug administration, which caused the drugs to flow into the ipsilateral upper extremity, and/or rapid drug administration. One patient experienced neck pain and headache, but recovered without any special treatment. The reason for the symptoms involved drugs flowing into the vertebral artery.

Previous studies have reported toxicity with systemic chemotherapy and hematological toxicity over grade 3 in $4-65 \%$ of patients $(15,16)$. Of the 53 patients included in the present study, no complications associated with the angiographic technique were observed. Hematological toxicity over grade 3 such as fever and without bleeding was observed in 6 cases $(11.3 \%)$. The patients were treated with human granulocyte colony-stimulating factor (human GCSF) and recovered. Nine patients had gastrointestinal symptoms including nausea, vomiting, diarrhea and stomachache. The patients were administered timely symptomatic treatment and recovered. Cardiovascular toxicity was not observed.
In conclusion, intra-arterial infusion chemotherapy is an effective treatment for local tumor control and tumor downstaging of LABC, thereby increasing the possibility for surgery. Low systemic toxicity and good patient compliance are also beneficial. However, severe complications may occur during treatment. Thus, controlling the pressure of the tourniquet and velocity of drug administration are crucial for reducing local complications .

\section{References}

1. Singletary SE, Allred C, Ashley P, et al: Revision of the American Joint Committee on Cancer staging system for breast cancer. J Clin Oncol 20: 3628-3636, 2002.

2. Kaufmann M, von Minckwitz G, Bear HD, et al: Recommendations from an international expert panel on the use of neoadjuvant (primary) systemic treatment of operable breast cancer: new perspectives 2006. Ann Oncol 18: 1927-1934, 2007.

3. Olson JE, Neuberg D, Pandya KJ, et al: The role of radiotherapy in the management of operable locally advanced breast carcinoma: results of a randomized trial by the Eastern Cooperative Oncology Group. Cancer 79: 1138-1149, 1997.

4. Murakami M, Kuroda Y, Nishimura S, et al: Intraarterial infusion chemotherapy and radiotherapy with or without surgery for patients with locally advanced or recurrent breast cancer. Am J Clin Oncol 24: 185-191, 2001.

5. Kitagawa K, Yamakado K, Nakatsuka A, et al: Preoperative transcatheter arterial infusion chemotherapy for locally advanced breast cancer (stageIIIb) for down-staging and increase of respectability. Eur J Radiol 43: 31-36, 2002.

6. Fiorentini G, Tsetis D, Bernardeschi P, et al: First-line intra-arterial chemotherapy (IAC) with epirubicin and mitoxantrone in locally advanced breast cancer. Anticancer Res 23: 4339-4345, 2003.

7. Pacetti P, Mambrini A, Paolucci R, et al: Intra-arterial chemotherapy: a safe treatment for elderly patients with locally advanced breast cancer. In Vivo 20: 761-764, 2006.

8. Shimamoto H, Takizawa K, Ogawa Y, et al: Clinical efficacy and value of redistributed subclavian arterial infusion chemotherapy for locally advanced breast cancer. Jpn J Radiol 29: 236-243, 2011.

9. Shenkier T, Weir L, Levine M, et al: Steering committee on clinical practice guidelines for the care and treatment of breast cancer. Clinical practice guidelines for the care and treatment of breast cancer: 15 . Treatment for women with stage III or locally advanced breast cancer. CMAJ 170: 983-994, 2004.

10. Huang EH, Tucker SL, Strom EA, et al: Predictors of locoregional recurrence in patients with locally advanced breast cancer treated with neoadjuvant chemotherapy, mastectomy, and radiotherapy. Int J Radiat Oncol Biol Phys 62: 351-357, 2005.

11. Fisher B, Bryant J, Wolmark N, et al: Effect of preoperative chemotherapy on the outcome of women with operable breast cancer. J Clin Oncol 16: 2672-2685, 1998.

12. Wolmark N, Wang J, Mamounas E, et al: Preoperative chemotherapy in patients with operable breast cancer: nine-year results from National surgical adjuvant breast and bowel project B-18. J Natl Cancer Inst Monogr 30: 96-102, 2001.

13. van der Hage JA, van de Velde CJ, Julien JP, et al: Preoperative chemotherapy in primary operable breast cancer: results from the European organization for Research and Treatment of Cancer trial 10902. J Clin Oncol 19: 4224-4237, 2001.

14. Mauriac L, MacGrogan G, Avril A, et al: Neoadjuvant chemotherapy for operable breast carcinoma larger than $3 \mathrm{~cm}$ : a unicentre randomized trial with a 124-month medial follow-up. Institut Bergonié Bordeaux Groupe Sein (IBBGS). Ann Oncol 10: 47-52, 1999.

15. Eniu A, Palmieri FM and Perez EA: Weekly administration of docetaxel and paclitaxel in metastatic or advanced breast cancer. Oncologist 10: 665-685, 2005.

16. Heller W, Mazhar D, Ward R, et al: Neoadjuvant 5-fluorouracil epirubicin and cyclophosphamide chemotherapy followed by docetaxel in refractory patients with locally advanced breast cancer. Oncol Rep 17: 253-259, 2007. 